\title{
تعليم اللغة العربية لأغراض أكاديمية في برنامج الكثف
}

\section{Muhammad Farih}

\section{Institut Keislaman Abdulah Faqih (INKAFA) Gresik}

farih_muhammad@yahoo.com

\section{مستخلص البحث}

البرامج لتعليم العربية لأغراض خاصة هي البرامج النوعية التى ينخرط فيها جمهور ذو طبيعة خاصة وحاجات محددة، تعليم اللغة لأغراض خاصة، مدخل لتعليم اللغة تستند كافة عناصره، من أهداف ومحتوى وطريقة تدريس، إلى الأسباب التي دفعت الدارسين لتعلم اللغة.

ويهدف هذا البحث وصف مشكلات تعليم اللغة العربية لأغراض أكاديمية في برنامج المكثف لتعليم اللغة العربية لدي طلبة كلية التربية الإسلامية بجامعة مولانا مالك إبراهيم بمالانج، وتقديم حلولها. و المنهج المستخدم في هذا البحث هو منهج الوصفي حيث يستخدمه الباحث لوصف مشكلات تعليم اللغة العربية لأغراض أكاديمية في برنامج المكثف لتعليم اللغة العربية لدي طلبة كلية التربية الإسلامية بجامعة مولانا مالك إبراهيم بمالانج وقد جمع الباحث البيانات باستعمال طريقة الملاحظة لعملية التعليم والمقابلة مع المدرس والدارس. وأبرز نتيجة البحث-على وجه العموم- هو أن العملية التعليمية تحتاج إلى تحسين في بعض الأمور مثل ضعف المدرس لمهارة وفن التدريس وعدم تنظيم التعليم الجيد واخلاف خلفية الطلاب وعدم موقف التعليم الجيد وعدم الكتاب الخاص عن تعليم اللغة العربية لأغراض أكادمية، وقلة وسائل التعلم وبتمد طريقة التعليم. وأن جودة تعليم اللغة العربية لأغراض أكاديمية تتعلق كثيرا بتوفر كفاءة المدرس وإيجاد موقف التعليم واختيار المادة واستخدام طرائق التعليم المناسب لحوائج الطلاب. الكلمات المفتاحية: تعليم اللغة العربية، أغراض أكاديمية 


\section{أساسيات البحث}

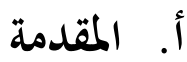

تنقسم برامج تعليم اللغة العربية لغير الناطقين بها بشكل عام إلى نوعين:

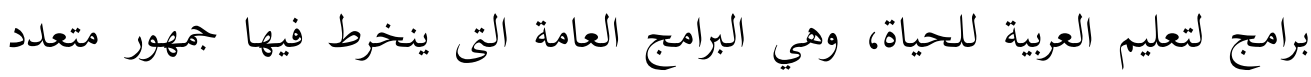

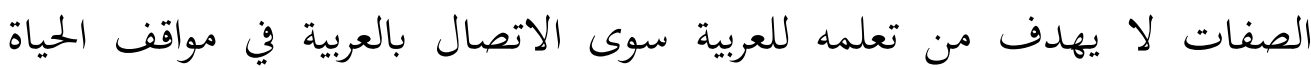

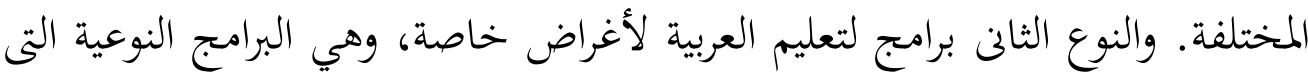

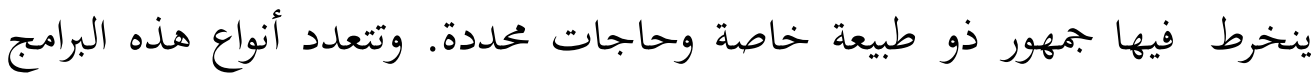

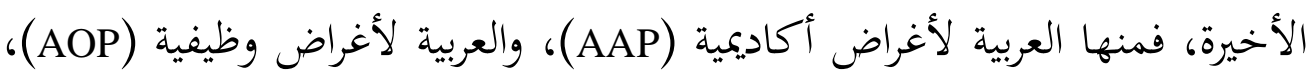

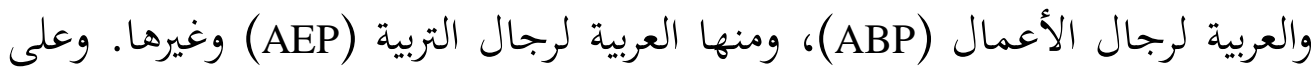

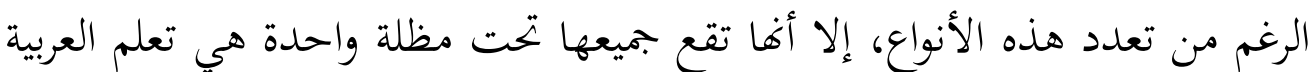

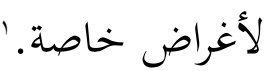

والجامعة مولانا مالك إبراهيم بمالانج تعتبر من أفضل الجامعات في إندونيسيا

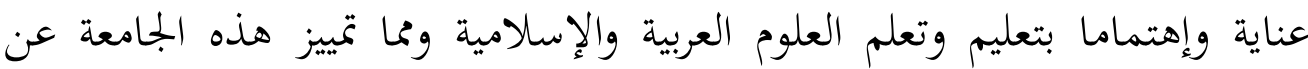

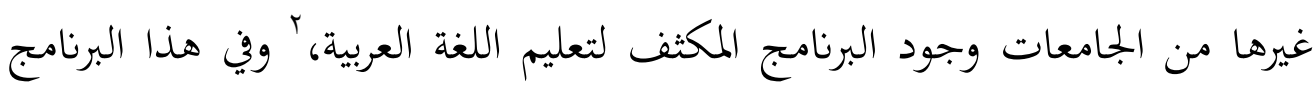

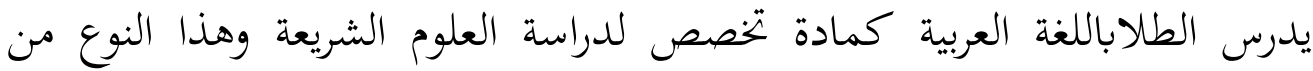
البرامج يطلق عليه: العربية لأغراض أكاديمية خاصة ولا شكك أن الواقع تعليم اللغة العربية يواجه مشكلات كثيرة حتى تتحقق الأهداف.

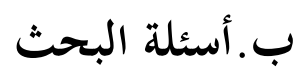

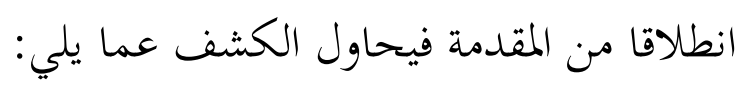

1. ما مشكلات تعليم اللغة العربية لأغراض أكاديمية في برنامج المكثف لمكف لتعليم اللغة

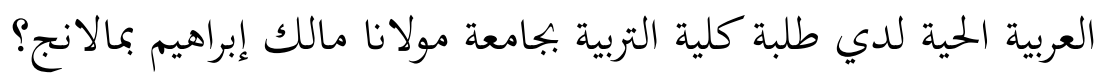

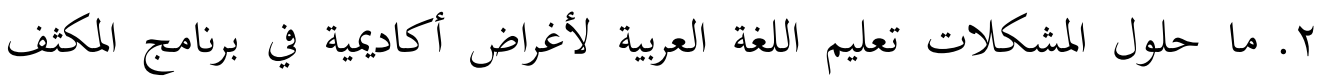
لتعليم اللغة العربية الحية لدي طلبة كلية التربية بجامعة مولانا مالك إبراهيم

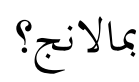

رشدي أممد طعيمة؛ محمود كامل الناقة. تعليم اللغة لأغراض خاصة: مفاهيمه ومنهجياته المشكلة ومسوغات الحركة

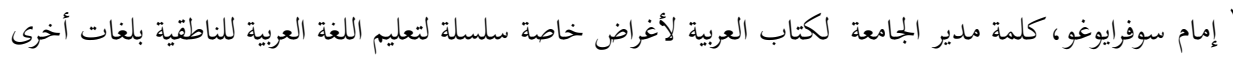




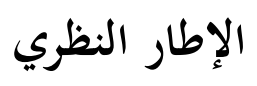

أ. تعليم اللغة العربية

\section{1 - مفهوم تعليم العربية لأغراض خاصة}

التعليم هو عامل من عوامل التربية وينحصر في إيصال المعلومات إلى

الذهن وصك حوافظ الشيء بمسائل العلوم والعلوم والفنون.ّ من هذا التعريف السابق يستعر فأن التعليم هو إعطاء المعلومات أوالمهارة وغيرها أو إلقاءها إلى هن

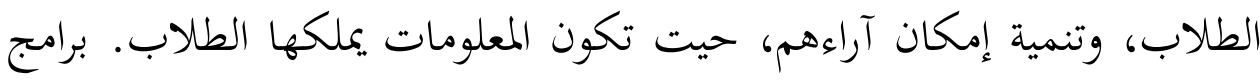
تعليم العربية للحياة وهي البرامج العامة التي هتم بتدريس العربية في سياقها التواصلي في مواقف الحياة المختلفة (في المطار-في المطعم-في السوق)، والنوع الثاني تعليم العربية لأغراض خاصة وهذا البرنامج موجه لفئات معينة من المتعلمين لهم حاجات خاصة كالعربية للدبلوماسيين والعربية لأغراض دينية وطبية وغيرها وتستند كافة عناصره من أهداف ومنهاج وطريقة تدريس إلى دوافع الطالب

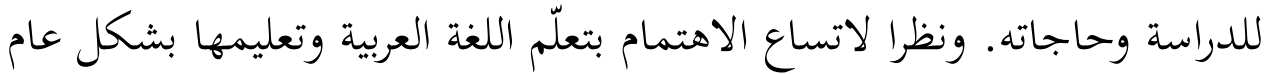
خاصّة بعد انتهاء الحرب الباردة بين أمريكا وروسيا فقد ذكر جلين نوردن Glenn

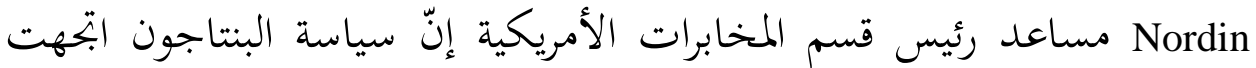
إلى تعليم وتعلم اللغات الأجنبية محددابجموعة من اللغات أهمها : الروسية تليها العربية فالصينية فالكورية فالفارسية وغيره.

ويرى طعيمة أن تعليم اللغة لأغراض خاصة شاع في بجال اللغة منذ عام 1971 ام ونشأ في البداية في الغرب على اللغة الإنجليزية ثم الفرنسية، ومفهوم

$$
\text { "كممود يونس وقاسم بكري. مجهول السنة، التربية والتعليم. ص: }
$$

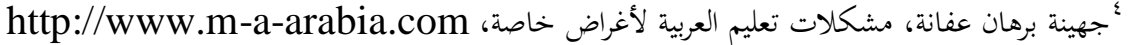

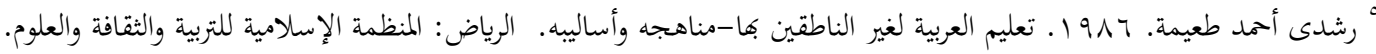


متعدد الأبعاد، بمثل ما اتعددت تعريفاته. ولعل أكثر التعريفات شيوعاً هو ما

يلي:

1- تعليم اللغة لأغراض خاصة، مدخل لتعليم اللغة تستند كافة عناصره، من أهداف ومحتوى وطريقة تدريس، إلى الأسباب التي دفعت الدارسين لتعلم

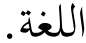

r-يميز كاتب آخر بين أربع خصائص أساسية (أومطلقة) وخاصيتين ثانويتين

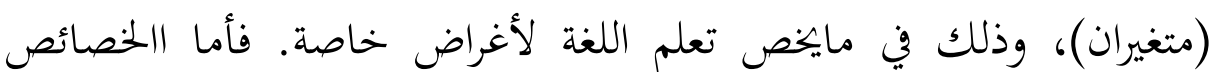

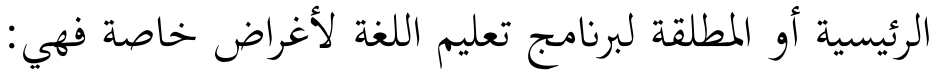

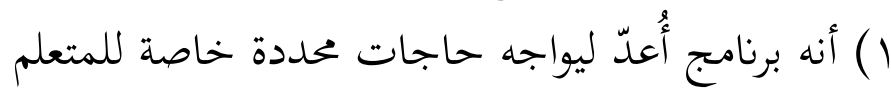

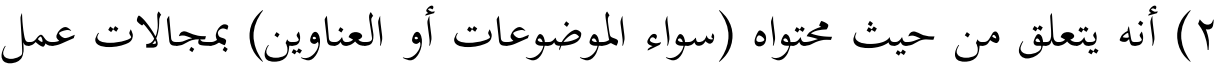

$$
\text { معينة أو مهن أو أنشطة حيث أنها }
$$

r) أنه يركز في اختيار اللغة على الأنشطة السابقة، سواء من حيث الثطة النحو،

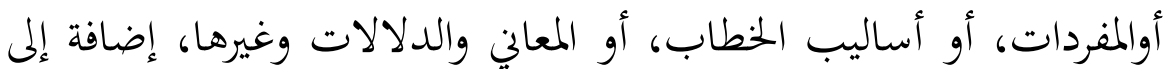

$$
\text { توظيف أساليب تحليل الخطاب. }
$$

$$
\text { ץ ؟ خصائص برامج يتناقض مع البرنامج العام لتعليم اللغة. }
$$

أما خصائص برامج تعليم الللغة العربية لأغراض خاصة التي قدمها جهينة

أ) البرنامج معدّ لتلبية حاجات محددة خاصة بالمتعلم

ب)هو يتعلق من حيث محتواه بمجالات معينة أو مهن أو أنشطة

ت)قد يتحدد البرنامج لتعليم مهارات معينة في مجال معين كالمحادثة للدبلوماسيين فقط

$$
\text { رشدي أممد طعيمة. المرجع السابق }
$$


ث) البرنامج يركز على اللغويات (معجميات وأساليب وأخيرا القواعد) والمهارات وأساليب الخطاب المناسبة للموضوع المراد دراسته

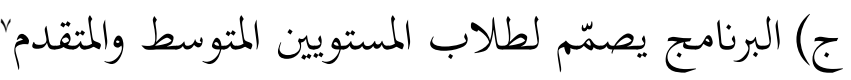

\section{ب. مشكلات التعليم}

مصطلح مشكلة في اللغة الإنجليزية مأخوذة من الكلمة "Problem" تعني بها المشكلة الأشياء التي لا يمكن حلها الذي يسبب المشاكل، والتعليم هو الجهد.

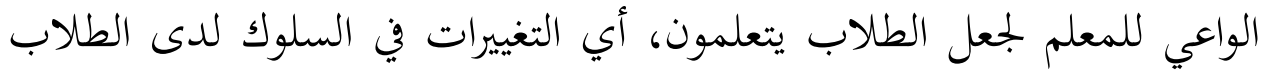
الذين يتعلمون ، حيث يتم الحصول على التغييرات بسبب قدرات جديدة تنطبق

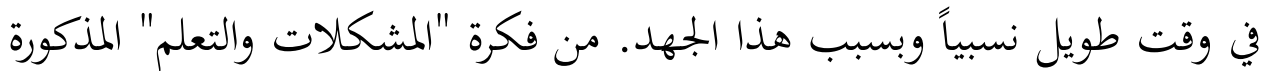
أعلاه، يمكن أن نستنتج أن فكرة "مشاكل التعيلم" هي مشكلة في عملية التعليم

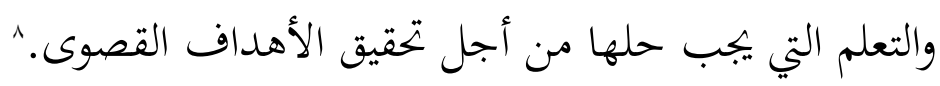

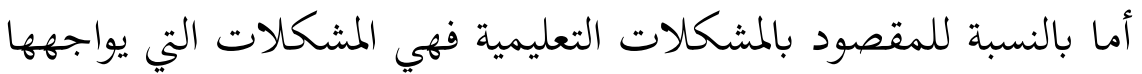
عالم التعليم، وخاصة دولة إندونيسيا. لقد أدى تطوير التعليم الذي تم منذ استقلال إندونيسيا إلى نتائج مثيرة للإعجاب، بحيث أن جودة الموارد البشرية

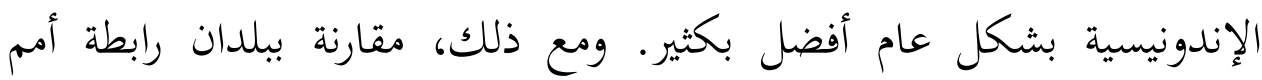
جنوب شرق آسيا، ما زلنا بعيدين عن التقدم، إن الجهود المبذولة لبناء الموارد البشرية ذات القدرة التنافسية العالية، والموجهة نهو العلم، والأخلاقية والثقافية ليست وظائف خفيفة نسبيًا. يحدث هذا بسبب عالم التعليم الذي لا نزال نواجه العديد من

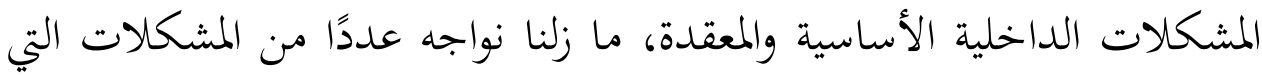
يتم تقييدها من التعليم الابتدائي إلى التعليم العالي، من المهم للغاية معالجة الجودة 
المتدنية على مستوى المدارس الابتدائية على الفور لأفما مؤثرة للغاية في التعليم

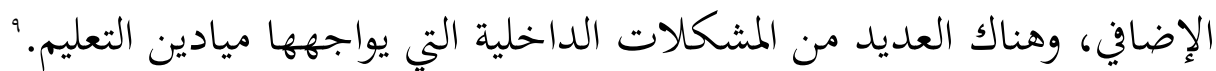

ومن مشكلات التعليم هي الجودة التعليمية في حالة إذا لم تصل النتائج

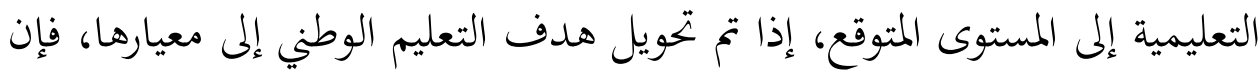

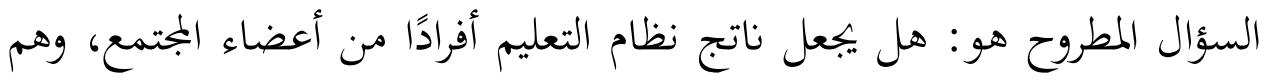

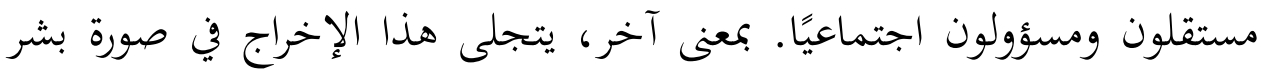

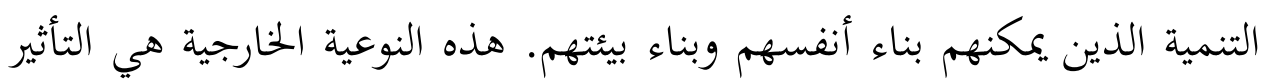
الحاد على الرغم من إدراك أن طبيعة المنتجات التي لها مثل هذه الخصائص

ليست حصيلة نظام التعليم.

\section{منهجية البحث}

المنهج المستخدم في هذا البحث هو المنهج الوصفي فبهذا المنهج يستطيع الباحث أن يقوم بوصف وكشف مشكلات تعليم اللغة العربية لأغراض أكاديمية في برنامج المكثف لتعليم اللغة العربية لدي طلبة كلية التربية الإسلامية بجامعة مولانا مالك إبراهيم الإسلامية الحكومية بمالانج من حيث الدارس والمدرس وطرائق التدريس والمادة التعليمية والبيئة العليمية وغير ذلك. وقد جمع الباحث البيانات المتعلقة بتعليم اللغة لأغراض أكاديمية في برنامج المكثف لتعليم اللغة العربية لدي الطلبة في كلية التربية الإسلامية بجامعة مولانا مالك إبراهيم الإسلامية الحكومية بمالانج بطريقة الوثائق والمقابلة والملاحظة الدقيقة.

\footnotetext{
${ }^{9}$ Eti Rochaety, dkk. 2006. Sistem Informasi Manajemen Pendidikan. Jakarta: PT Bumi Aksara, Hal: 64-65

${ }^{10}$ Umar Tirtarahardja dan La Sulo. 2005. Pengantar Pendidikan. Jakarta: PT Rineka Cipta. Hal: $232-233$
} 


\section{عرض البيانات ومناقشته \\ أ. مشكلات تعليم اللغة العربية لأغراض أكاديمية}

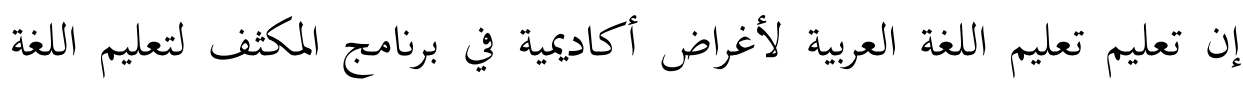

العربية لدي الطلبة كلية التربية الإسلامية متعلق بالعوامل المهمة منها تعليم المهارات

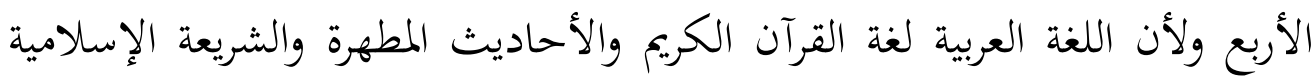
وهي في نفس الوقت ترتبط بالمواد التي تدرس في المدرسة كما توجد اللغة في المسابقة مثل الخطابة والمسرحية والأغاني. وتتعلق تعلم اللغة العربية لدي الطلبة بتحقيق التواصل الاجتماعي مع المدرسين والزملاء من الشعوب المتفرقة ومن الدول العربية كالمملكة السعودية ومصر وغيرها. رغم أنه من تلك الأماني هنالك مشكلات تعليم اللغة العربية لأغراض خاصة

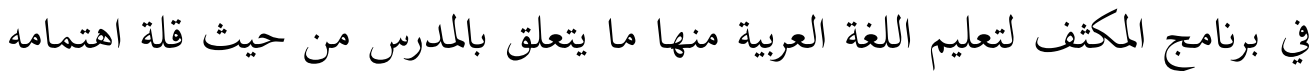
بالمحاضرة وما يتعلق بالدارس حيث امتلئت الدروس وكثرت الواجبات الأخرى وهو أيضا تكاسل، وهذه المشكلات مما يؤدي إلى فشال تعليم اللغة العربية. أما المشكلات التي تتعلق بلمواد التعليمية فهي عدم المادة التي تلائم مستويات

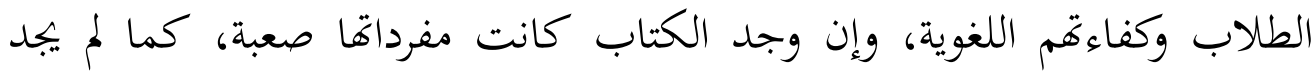

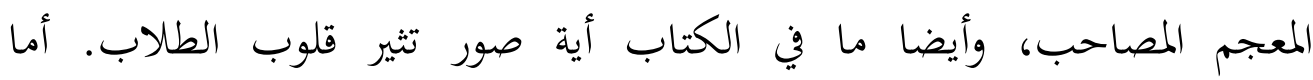

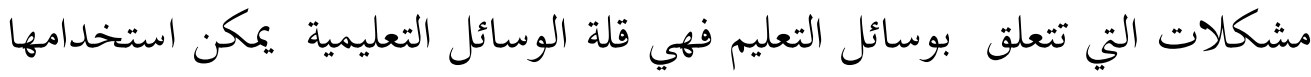

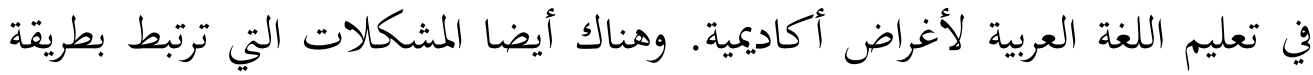
التعليم حيث اعتمد المدرس على طريقة الندريس المملة حتى لا يقبلها الدارس. ب. حلول مشكلات تعليم اللغة العربية لأغراض أكاديمية

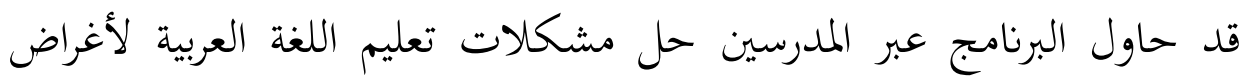

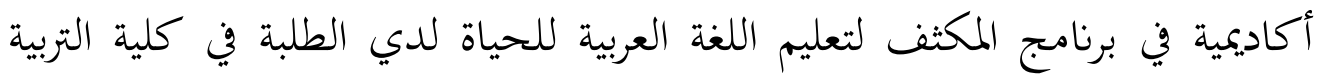
الإسلامية معتمدا على تنفيذ التعليم المذكور أدناها: 1- - أن من أهداف تعليم اللغة العربية تحقيق التعليم لأغراض أكاديمية 
Y- أن عملية التعليم لا بد أن تتم بتحقيق أهدافها التي قد قرها البرنامج

r- أن البرنامج المكثف لتعليم العربية يطلب من المدرس الكفاءة التعليمية

لذا، المحاولات التي يجب على مدرس البرنامج المكثف لتعليم اللغة العربية بكلية

التربية الإسلامية بجامعة مولانا مالك إبراهيم مالانج قيامها في حل المشكلات

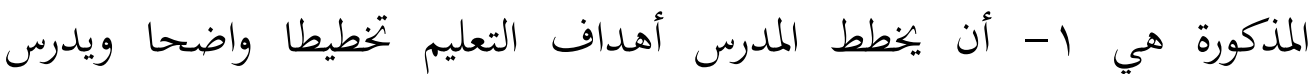
مستويات الطلاب اللغوية، ب-أن يعرف المدرس على الطلاب قبل بداية الدراسة عن أهداف التعليم التي يراد تحقيقها جماعيا، وأيضا على موظف البرنامج المكثف أن بعربـ

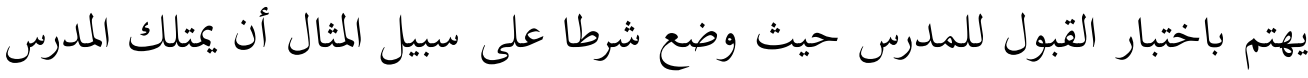
شرطا أساسيا أن يكون كفاءة التدريس ومهارة اللغة العربية. ومن أجل ترقية كفاءة المدرس في البرنامج المكثف بكلية التربية الإسلامية جامعة مولانا مالك إبراهيم الإسلامية الحكومية على المدرس اشتراك البرنامج لترقية المهنة مثل الدورة التدريبية عن اللغة العربية، والحضور في المحاضرة في تمام الوقت، ذلك لأنه مما يساعد نجاح عملية التعليم. أما المحاولات يمكن المدرس قيامها في حل المشكلات المذكورة فهي: أن يهتم المدرس بالمحاضرة، إن تعذر فينبغي أن يخبر عن عذره إلى إدارة قسم شؤون التوظيف. إلى جانب ذلك بإمكان المدرس المتعذر أن يطلب من الطلبة بالمناقشة وغيرها.

إضافة إلى ذلك أن يفهم المدرس مستويات الطلاب اللغوية ويميزوها ويجوز له تقسيم الطلبة المتقدمين بين الطلاب الضعاف. وأيضا أن يعطي المدرس الوظيفة الإضافية للطلاب مثل قراءة النصوص القرآية ويشجعهم على حب اللغة العربية حبا جما عبر أي طريقة ممكنة. وعند إجراء عملية التعليم والتعلم تدور المادة التعليمية (مضمون) دورا هاما، أما إذا كانت المادة مناسبة لكفاءة الطلاب فتحقق الأهداف التعليمية غير أن المادة التعليمية تحتاج أيضا إلى الوقت المتوفر. ولا يجوز للمدرس أن يتكلم غير اللغة العربية وأن يكون أسوة لغوية في كل وقت وحين. وكذلك الوسائل المعينة هي محا يحقق 
أهداف التعليم المرجوة، وبالوسائل التعليمية الحديثة مثلا تثير نفوس الطلاب في

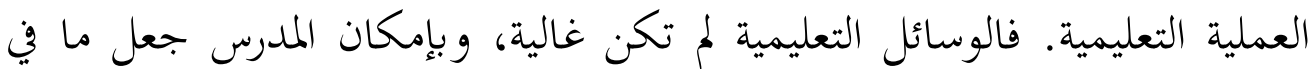

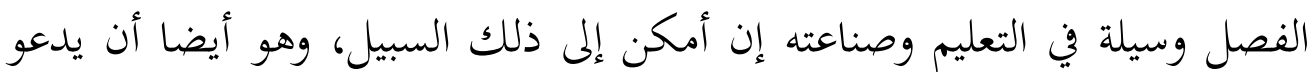
الطلاب إلى التعلم خارج الفصل كي يجذب جو التعليم رغبة الطلاب في التعلم. وكان للطريقة دور هام في تحقيق أهداف التعليم، فالمحاولة التي يقوم هما المدرس المابل في حل المشكلة التعليمية هي: مطالعة الكتب التي تتعلق بفن التدريس، واشتراك التدريب في ترقية فن تعليم اللغة العربية، واختيار طريقة التعليم أيضا مما يعين المدرس المئس

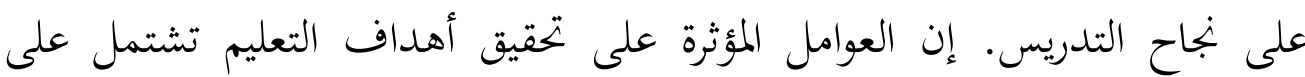

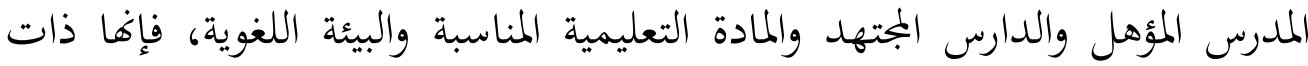
علاقة تأثير وتأثر. كلما ازداد تحسين إجراء عملية التعليم قد تحققت أهداف التعليم.

ومن البيانات التي عرضها الباحث يمكن تلخيص أهم نتائجها فيما يلي: إن من العوامل المؤثرة على عدم إجراء عملية التعليم الجيد هو المدرس نفسها. لذا، إذا أراد المدرس نجاح التعليم، فيجب عليه أن يكون أسوة حسنة لنفسه ولغيره

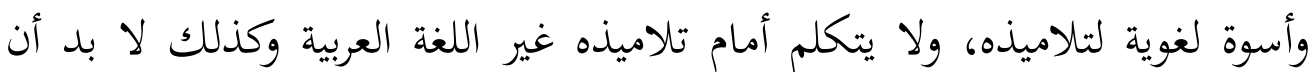
يخضر المحاضرة في تقام الوقت، ولا يجوز له التأخر بل هو غائب عن المحاضرة بدون

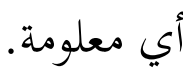

ونظرا لأهمية تعليم اللغة العربية لأغراض أكادمية فعلى المدرس الاهامام بالأمور

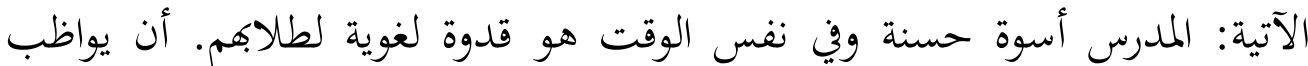

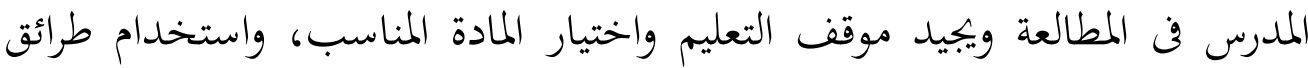
التعليم المتنوعة، وكل ذلك من العناصر الرئيسة في إنجاح العملية التعليمية. 


\section{الاختتام}

أ. ألمُلاصة

بعد أن عرض الباحث البيانات التي تتعلق بتعليم اللغة العربية لأغراض

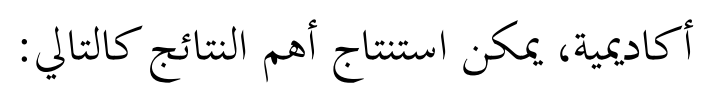

1- إن أهداف التعليم غير متحققة لأسباب منها ضعف المدرس لمهارة وفن

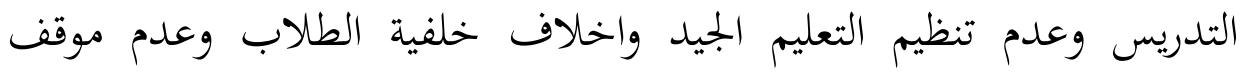
التعليم الجيد، وعدم الكتاب لخاص عن تعليم اللغة العربية لأغراض أكادمية، وقلة وسائل التعلم وبتحمد طريقة التعليم.

r- إن جودة تعليم اللغة العربية لأغراض أكاديمية متعلقة بتوفر كفاءة المدرس وإيجاد موقف التعليم واختيار المادة واستخدام طرائق التعليم المناسب لحوائج الطلاب.

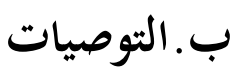

على المؤسسة التربوية أن هتتم بترقية جودة تعليم اللغة العربية لأغراض أكاديمية عبر ترقية كفاءة المدرس وإيجادة موقف التعليم واختيار المادة واستخدام

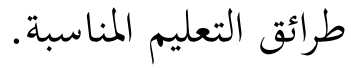




\section{قائمة المصادر والمراجع}

إمام سوفرايوغو. كلمة ملدير الجامعة لكتاب العربية لأغراض خاصة. سلسلة لتعليم الغة العربية للناطقية بلغة أخرى.

محمود يونس وقاسم بكري. مجهول السنة. التربية والتعليم.

رشدي أحمد طعيمة؛ محمود كامل الناقة. تعليم اللغة لأغراض خاصة: مغاهيمه ومنهجياته المشكلة ومسوغات الحركة.

http://www.m-a-.جهينة برهان عفانة. مشكلات تعليم العربية لوغراض خاصة arabia.com

رشدى أحمد طعيمة. 7 ا9 ا ـ تعليم العببية لغير الناطقين جها-مناهجه وأسالبه. الرياض: المنظمة الإسلامية للتربية والثقافة والعلوم

Debdikbud. 2002. Kamus Besar Bahasa Indonesia. Jakarta: Bulan Bintang.

Mochtar Buchori. 1994. Spektrum Problematika Pendidikan di Indonesia. Yogyakarka: Tiara Wacana Yogya.

Eti Rochaety, dkk. 2006. Sistem Informasi Manajemen Pendidikan. Jakarta: PT Bumi Aksara.

Umar Tirta Rahardja dan La Sulo. 2005. Pengantar Pendidikan. Jakarta: PT Rineka Cipta 